\title{
Evolución de los conjuntos urbanos y su influencia en el crecimiento poblacional y el desarrollo de los espacios periurbanos en Calimaya, Estado de México, de 1990 a 2015
}

\author{
María Lourdes García-González, Fermín Carreño-Meléndez \\ y Alfonso Mejía-Modesto
}

Universidad Autónoma del Estado de México

\section{Resumen}

Este trabajo tiene el propósito de analizar la evolución e influencia de la producción habitacional del sector privado en la expansión de la urbanización y crecimiento de la población en Calimaya, Estado de México, de 1990 a 2015, mediante el uso de técnicas cualitativas y cuantitativas, tomando como base información de la Secretaría de Desarrollo Urbano estatal y municipal, INEGI y los SIG. Los datos estadísticos, cartográficos, la observación y análisis del proceso de producción habitacional a través de conjuntos urbanos en Calimaya muestran, por un lado, su tendencia a extenderse sin control en las localidades de Calimaya de Díaz González, San Andrés Ocotlán y la Concepción Coatipac, sobre las áreas de vocación agrícola que las circundan, conformando un espacio periurbano residencial desordenado y disperso, y por otro, el crecimiento acelerado de la población y sus posibles impactos sociales y ambientales derivados de estos.

Palabras clave: Conjuntos urbanos, crecimiento poblacional y espacios periurbanos.

\section{Abstract}

Evolution of urban complexes and their influence on population growth and development of the peri-urban spaces in Calimaya, State of Mexico, from 1990 to 2015

This paper aims to show the evolution and influence of housing production in the private sector in expanding urbanization and population growth in Calimaya, State of Mexico, form 1990 to 2015, using qualitative and quantitative techniques, taking basis information from the Secretariat of state and Municipal Urban Development, INEGI and GIS. Statistical, cartographic data, observation and analysis of the process of housing production through urban complexes in Calimaya show on the hand its tendency to spread out of control in the towns of Calimaya Diaz Gonzalez, San Andres Ocotlan and the Concepción Coatipac, on the areas suitable for agriculture that surround forming a disorderly and scattered suburban residential space, and secondly, the rapid population growth and its potential social and environmental impacts of these.

Kew words: Urban complexes, population growth and peri-urban space. 


\section{INTRODUCCIÓN}

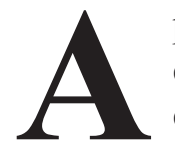

partir de la década de los noventa pero principalmente en la década del 2000, producto de la relación físico-funcional con la ciudad de Toluca, el fenómeno de conurbación y metropolización en torno a ésta, comenzaron a expandirse en el municipio de Calimaya nuevas formas de uso del espacio rural, basadas en actividades de producción habitacional en el esquema de conjuntos urbanos: residenciales, medios y de interés social, en urbanizaciones cerradas y abiertas las cuales dan cuenta de nuevas formas de apropiación del espacio y mercantilización del espacio rural, ligadas a nuevas necesidades de los habitantes urbanos.

En este sentido, la expansión residencial en los espacios rurales de la periferia de tres localidades del municipio de Calimaya (Calimaya de Díaz González, San Andrés Ocotlán y La Concepción Coatipac) y el rápido crecimiento de la población del municipio, es un tema de interés para la investigación urbana, ya que el proceso de expansión se ha estudiado para las ciudades grandes y medias como un fenómeno propio de estas zonas; sin embargo, en años recientes este proceso se está presentando en ciudades pequeñas y comunidades rurales, convirtiéndose en un ejemplo de hiperurbanización desordenada y dispersa, que requiere de estudios que la aborden desde diversos enfoques que sirvan de base para la formulación de políticas públicas en materia de desarrollo urbano y ordenamiento del territorio.

Este trabajo tiene el propósito de analizar la evolución de la producción habitacional de los conjuntos urbanos, en la expansión residencial; conformando espacios periurbanos y acelerando el crecimiento de la población de Calimaya, considerando las transformaciones de los espacios de la periferia rural, de 1990 a 2015.

De esta manera, tomando como base las estadísticas de conjuntos urbanos autorizados por la Secretaría de Desarrollo Urbano del Estado de México y la dirección de Desarrollo Urbano de Calimaya, censos y conteos de población y vivienda del periodo de estudio, y trabajo de campo, se realizó el análisis y cartografía que permitió, a través de la interpretación de datos y mapas, dar respuesta a las preguntas sobre cómo, dónde y en qué condiciones se ha producido vivienda para la población de la zona metropolitana de Toluca y su influencia en el crecimiento de la población del municipio. 


\section{REFERENTES TEÓRICOS}

El análisis de los procesos de urbanización en los países en desarrollo ha sido estructurado tradicionalmente en torno a dicotomías como; rural-urbano, tradicional-moderno, formal e informal. En este marco, la interface periurbana es entendida como la pérdida de valores "rurales" (pérdida de suelo fértil, paisajes naturales, etc.) o déficit de atributos "urbanos" (baja densidad, falta de acceso, ausencia de servicios e infraestructura, etc.) (Adell, 1999).

Estas manifestaciones territoriales del crecimiento y expansión de las grandes ciudades, dieron lugar a la elaboración de teorías generales sobre la emergencia de un nuevo sistema de poblamiento. Inicialmente se planteaba la existencia de un "retorno al campo", posteriormente conceptuado bajo el término contraurbanización (Berry, 1976), caracterizada principalmente por los nuevos flujos poblacionales rurales en el contexto de las actividades inmobiliarias.

Otros autores señalaban el "renacimiento" de las zonas no metropolitanas (Kayser, 1990; Kayser, 1993); también se insistía en una repartición menos concentrada de la población de procesos tales como: la desconcentración y redistribución demográfica, así como la reestructuración regional de la economía y el espacio (Champion, 1992 citado en Ávila, 2001).

Respecto a los factores que definen los diferentes enfoques teóricos, Zarate (1991), menciona que entre otros elementos que explican esta estructura a través de modelos de organización y función del espacio urbano, se tiene la distribución en el territorio de los diversos paisajes y usos del suelo con una estructura determinada por un centro, áreas residenciales, zonas industriales y el espacio periurbano. Allen (2003), considera que estos procesos coinciden con una fase de transición de una sociedad industrial a una post-industrial, en la cual los espacios rurales pueden ser "favorecidos" y donde se puede observar una urbanización difusa, e identificar los espacios rururbanos o periurbanos, aseveraciones insuficientes para aprehender la naturaleza cambiante de la interface urbana.

Respecto a la localización de nuevas formas de urbanización como quintas y villas alrededor de los centros urbanos son considerados como un fenómeno que ya existía en las ciudades romanas y posteriormente en las renacentistas. Fue en el transcurso del siglo XIX, cuando las transformaciones de la periferia urbana se tornaron notables y dieron origen al estudio del periurbano como objeto de investigación. En ello influyeron los intensos procesos de suburbanización en las ciudades anglosajonas en 
el contexto de la Segunda Revolución Industrial, la accesibilidad generada por el ferrocarril y el automóvil, el establecimiento del telégrafo y el teléfono (Capel, 1994).

Sin embargo, durante la primera mitad del siglo XX, este proceso se consolidó debido a una descentralización mayor de las actividades productivas de la ciudad, la construcción de autopistas y el acceso al crédito hipotecario por parte de diversas clases sociales, las cuales generaron la denominada "ciudad difusa" alrededor de las décadas de los 50 y 60. Posteriormente los procesos que siguieron a éste periodo se caracterizaron por el avance de diversos servicios urbanos y tecnologías fuera de la ciudad, redes de autopistas y una revalorización social del "medio natural" por parte de los sectores económicamente acomodados, fenómenos que intensifican las transformaciones del periurbano, de tal manera que, en la medida que la urbanización avanza sobre el ámbito rural, origina conceptos nuevos que dan cuenta de nuevas formas de ocupación y re-organización de estos espacios (Puebla, 2004).

En los países anglosajones el periurbano se ha formado como el lugar de residencia de las clases acomodadas, donde su configuración es condicionada de manera importante por el modelo de ciudad-jardín con determinadas pautas de planeación (Garay, 2001). Para los países latinoamericanos el periurbano es principalmente habitacional, donde se expresa la diferenciación territorial en cuanto a las formas de apropiación y uso del espacio, en el cual conviven al mismo tiempo barrios de población de altos ingresos colindando con zonas marginales, donde coexisten actividades informales con las prácticas agrícolas, configurando el avance de la urbanización a lo largo de los ejes de salida de las ciudades por poblamientos difusos, que afecta tanto a zonas agrícolas de alto y bajo valor productivo, espacios que pueden presentar problemáticas sociales y ambientales agudas (Puebla, 2004 y Ávila, 2009).

En México, Aguilar (2006) considera que en las periferias, las áreas residenciales han sufrido cambios profundos referidos a la construcción de complejos de vivienda unifamiliar dirigidos a diversos niveles sociales, así como las grandes obras de infraestructura como aeropuertos o principales vías de comunicación, las cuales facilitan el desarrollo de actividades productivas que, en su conjunto, atraen la ubicación de toda clase de usos de suelo.

En esta transformación del periurbano y en un contexto de formación de nuevas centralidades, surge la construcción masiva de viviendas con características como la cerca perimetral y control de sus accesos, bajo los 
conceptos de seguridad, exclusividad y contacto con la naturaleza, cuyo objetivo es ofrecer una mayor calidad de vida a sus residentes, cuya denominación depende de sus características y localización; guetos de lujo o enclaves fortificados en Brasil; gated community en Estados Unidos; fraccionamientos o desarrollos cerrados en México, el cual varía según las localidades (Cabrales y Canosa, 2001).

En este marco, el estudio del periurbano considera el planteamiento de un territorio complejo que expresa un estado de interface entre dos zonas aparentemente bien diferenciadas: el campo y la ciudad, de difícil delimitación y definición conceptual. En cuanto a objeto de investigación, un territorio problemático, en situación de transición, en permanente transformación o con expectativas de ser transformado que al paso del tiempo, se extiende, se relocaliza, heterogéneo en los usos del suelo, nombrado de diferentes maneras: la periferia urbana, el rur-urbano, la "ciudad difusa", la frontera campo-ciudad, la "ciudad dispersa", territorios de borde, borde urbano/periurbano, el contorno de la ciudad, exurbia, etc., espacio que se define por la indefinición: no es campo, ni ciudad (Barsky, 2005).

Por otro lado, la incorporación del sector privado en la producción habitacional impulsada por las instituciones públicas en la primera década del siglo XX fue determinante en el desarrollo del espacio periurbano. Entendida la producción habitacional como el proceso relacionado con la planeación, promoción, construcción y distribución de los espacios habitacionales, incluido su entorno físico próximo en el territorio. Duhau (2008) nombró a este sector como los nuevos productores del espacio habitable.

\section{URBANIZACIÓN Y POLÍTICA HABITACIONAL}

Los cambios en la política habitacional de México hasta su situación actual nos conduce a la segunda mitad de los años noventa, como consecuencia de los cambios en la política económica del país, en la que desaparecen las soluciones habitacionales urbanas (lotes con servicios, pie de casa, piso firme y de vivienda entre otros) y de vivienda rural para los grupos más vulnerables de menor ingreso, la transformación de los organismos nacionales y estatales de vivienda de las últimas décadas han experimentado cambios sustantivos en el financiamiento y producción de vivienda de interés social, así como por el crecimiento acelerado de las empresas desarrolladoras que han tomado casi en su totalidad el proceso de producción de vivienda.

El cambio de los organismos públicos, de promotores a financieros se generó por dos procesos: el primero se refiere a la reordenación y concentración de la demanda en el sector de trabajadores formales con un ingreso 
de tres a diez veces el salario mínimo (vsm) equivalente a cerca de 25 a 30 por ciento de la demanda de vivienda urbana y el segundo corresponde al traslado de las funciones de incorporación del suelo a usos urbanos, de urbanización y producción, promoción y venta de viviendas a los desarrolladores inmobiliarios privados. Con esto, el mercado habitacional de interés social que era guiado por la demanda y necesidades a través del estado se ha transformado, a otro donde son los oferentes quienes lo determinan con la complacencia del gobierno (Iracheta y Pedrotti, 2013).

Así, la concepción del papel del Estado en la política de vivienda a nivel nacional cambio de benefactor a regulador y facilitador de negocios. Los cambios en las políticas públicas han implicado una menor intervención en materia de planeación territorial y producción de vivienda, en temas centrales como: la localización de los conjuntos urbanos, las soluciones habitacionales para los sectores más pobres, la construcción y mantenimiento de la vivienda, entre otras (Puebla, 2004).

En este sentido, la dirección de la política habitacional se ha concretado en alcanzar las metas de otorgamiento de créditos hipotecarios, de construcción de casas, así como en desarrollar estrategias financieras para ampliar esta producción. Por tanto, el Estado mexicano ha asumido la función de gestión-administración de mecanismos, incentivos e instrumentos que faciliten y garanticen la ejecución de las iniciativas y proyectos de vivienda del sector privado para que se cumplan las metas de financiamiento y producción de casas y otorgamiento de créditos hipotecarios a los trabajadores, en detrimento del ordenamiento de las ciudades y el equilibrio ambiental.

\section{Bases normativas del conjunto urbano en el Estado de México}

La legislación en materia de urbanización en el Estado de México ha cambiado de acuerdo con su evolución, principalmente en relación a la autorización de la subdivisión y fraccionamiento del suelo y la normatividad de vivienda, la cual se concentra actualmente en el Libro V del Código Administrativo del Estado de México (CAEM) y su reglamento, los cuales contienen las normas en relación con el ordenamiento territorial de los asentamientos humanos y el desarrollo urbano de los centros de población, y en el Código Financiero del Estado de México y municipios. Del cual destaca en el proceso de urbanización y producción de vivienda un fuerte enfoque administrativo y financiero.

A partir del artículo 5.40 en adelante en el que se define el conjunto urbano, se establecen los procedimientos para la producción de estos (tipolo- 
gía, normas generales, derechos y obligaciones del desarrollador, derechos y obligaciones de los municipios donde se construyen los conjuntos, los permisos y licencias, etc.), los cuales se norman con más detalle en su respectivo reglamento. El artículo 5.44 hace referencia a las obras de infraestructura, urbanización y equipamiento urbano al interior del conjunto, pero no se observan referencias específicas sobre las obras externas que conectan al conjunto urbano con la ciudad o el centro urbano donde se localiza.

El artículo 5.5.9 establece los lineamientos y procedimientos para la autorización de los conjuntos urbanos, cuyos criterios refieren la coherencia respecto al uso del suelo establecido en el plan de desarrollo urbano municipal, los cuales parecen ser insuficientes al no considerar las tendencias de crecimiento de la mancha urbana para la localización del conjunto dentro del municipio.

Por otro lado, el reglamento del Libro V, artículo 40, fracción II, establece que para la autorización de conjuntos urbanos, su dimensión y densidad estarán en función de la disponibilidad de agua potable, presenta una inconsistencia, ya que considera la disponibilidad como la capacidad institucional de proveer el líquido y no considera las condiciones de los acuíferos de donde se extrae los cuales pueden encontrarse en crisis, como es el caso del Valle de Toluca.

Posteriormente en el año 2006, se formó la Comisión Estatal de Desarrollo Urbano y Vivienda como órgano técnico de coordinación interinstitucional, presidida por funcionarios estatales, Comisión Federal de Electricidad (CFE) y los municipios respectivos, con funciones sustantivas estipuladas en el artículo 5.12 del libro V del CAEM, donde se integran las opiniones técnicas para otorgar la Constancia de Viabilidad al proyecto de un conjunto urbano. Procedimiento clave en la autorización, ya que contiene los siguientes dictámenes según el artículo 45 del reglamento del Libro V:

- De existencia y dotación de agua potable para el desarrollo.

- De protección civil, en materia de seguridad del suelo y riesgo.

- De evaluación de impacto ambiental.

- De incorporación e impacto vial.

- En su caso, dictámenes de instancias federales y estatales por el uso de derecho de vía.

- Certificación de clave catastral y secuencia registral que emita el Registro Público de la Propiedad.

- Constancia de capacidad de suministro de energía eléctrica.

- Licencia de uso del suelo. 
Se puede señalar que aun cuando se trata de temas relevantes para emitir una opinión fundada de apertura y crecimiento urbano, asuntos como: la consolidación de la mancha urbana, congruencia del proyecto con las tendencias de crecimiento físico y demográfico, la ubicación respecto a vialidades conectoras de forma ordenada en relación a la urbanización existente y condiciones ambientales, no quedan claros. El análisis de la localización de cada conjunto urbano en Calimaya, como se nota más adelante, confirma que no obstante la cantidad de dictámenes y constancias, la realidad es que los conjuntos urbanos autorizados y construidos hacen ciudad.

\section{El CONJUNTO URBANO RESPONSABLE DE LA FORMACión DEL PERIURbano RESIDENCIAL EN CALIMAYa}

Como ya se mencionó, la política federal y estatal de vivienda se centra en alcanzar metas de producción de vivienda y desarrollar estrategias financieras para ampliar la producción de casas. Para alcanzar dichas metas, en el Estado de México la producción habitacional ha sido impulsada por la creación de la figura del conjunto urbano, por medio de la cual se ha otorgado la función de desarrollo y oferta de vivienda de interés social a las empresas privadas, donde la localización de los conjuntos urbanos y los impactos (territoriales, sociales y ambientales) son menos importantes, (Iracheta y Pedrotti, 2013).

Para alcanzar estas metas, el interés de los desarrolladores ha sido la adquisición de suelo periférico, cada vez más lejano a los centros de población debido a su bajo costo, incurriendo con mayor frecuencia en la compra de suelo ejidal, que los planes contemplan como no programado para expansión urbana, o si el suelo adquirido tiene un uso distinto al habitacional, se hacen los cambios de uso de para la construcción de conjuntos urbanos.

En este contexto, el patrón de urbanización que ha generado la producción de conjuntos urbanos en Calimaya, podría denominarse según Duhau (2008), como modelo de ínsula, en la medida que la producción de estructuras urbanas sólo se vincula a su entorno y a las ciudades por medio de alguna vialidad primaria; implica que los nuevos habitantes de estos conjuntos urbanos se enfrenten a los aspectos negativos como: el aislamiento de su hábitat, la lejanía del trabajo, de la escuela, de los servicios de salud, de mercados y financieros, servicios que la ciudad ofrece, además de la traza urbana y distribución de los usos de suelo en el interior según la lógica funcionalista bajo la cual se construyen. 


\section{Materiales y MÉtodos}

\section{Área de estudio}

El municipio de Calimaya ocupa el tercer lugar de los municipios de la Zona Metropolitana de Toluca y el quinto lugar del estado de México en cuanto a su extensión territorial que es de $103.4 \mathrm{~km}^{2}$. Se localiza al sur de Toluca y se ubica geográficamente en los paralelos $99^{\circ} 37^{\prime} 02^{\prime \prime}$ de longitud oeste y en los $19^{\circ} 10^{\prime} 25^{\prime}$ " de latitud norte. Limita al norte con los municipios de Toluca, Metepec, Mexicaltzingo y Chapultepec; al oriente con Tianguistenco y San Antonio la Isla; al occidente con Toluca; y al sur con Tenango del Valle y Santa María Rayón (Figura 1). El territorio ocupa un amplio plano inclinado que al oeste se encuentra la parte más alta, a los 4,578 msnm, que baja hasta el terreno plano de la parte oriental, a una altura de 2,600 msnm. La cabecera municipal tiene una altitud media de 2 $680 \mathrm{msnm}$.

\section{Metodología}

La evolución de los conjuntos urbanos en el desarrollo de los espacios periurbanos y su influencia en el crecimiento de la población en Calimaya se aborda desde un enfoque de investigación cualitativo, cuyo alcance es describir la evolución de la producción habitacional del sector privado bajo la figura de conjuntos urbanos, y explicar su influencia en el crecimiento de la población en el municipio. Se trata de una investigación no experimental.

El trabajo se desarrolló en tres fases:

1. Para recopilar la información necesaria, se consultó el archivo de autorizaciones de conjuntos urbanos por la Secretaría de Desarrollo Urbano del Estado de México publicadas oficialmente en las Gacetas de Gobierno del Estado de México y la Dirección de Desarrollo Urbano del municipio, identificando las autorizaciones de estos conjuntos de uso habitacional en la zona de estudio de 1990 a 2010. Los datos levantados fueron: nombre y dirección del conjunto urbano, fecha de autorización, superficie, número de viviendas edificadas, número de lotes, tipo de conjunto urbano. Además, se consultaron otras fuentes de información como: censos y conteos de población del INEGI del periodo de estudio, cartografía, Planes de Desarrollo Urbano del municipio, Plan Regional de Desarrollo Urbano de la Región Toluca vigente y la utilización del programa Mapa Digital. 


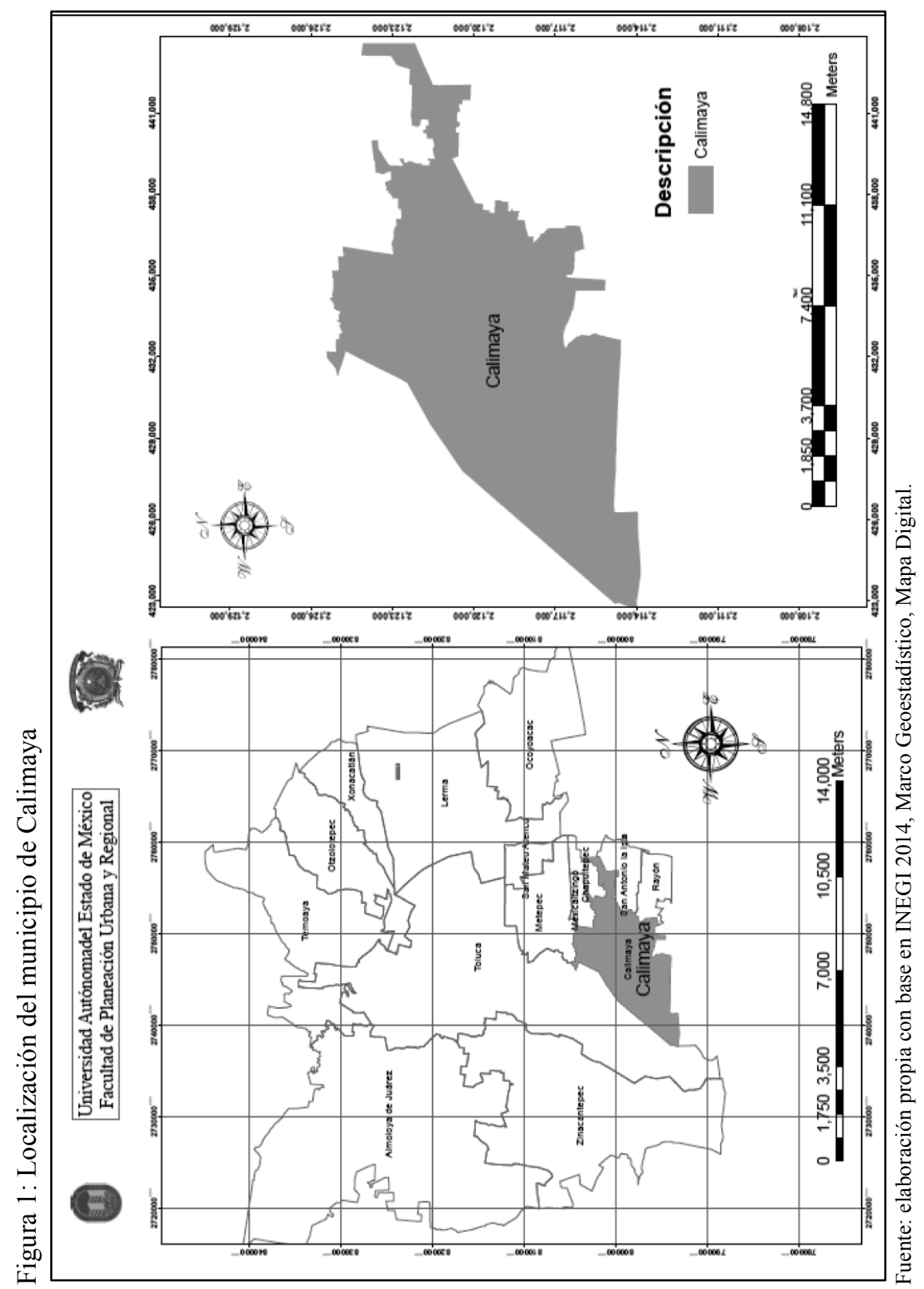


2. El trabajo de campo se llevó a cabo mediante un recorrido en la zona de estudio, con la ayuda del GPS para la identificación y geo referencia de los conjuntos urbanos habitacionales, para verificar la información obtenida en los registros estatal y municipal.

3. Con la información inicial, se elaboró la base de datos con la cual se realizaron diversos cálculos estadísticos básicos que permitieron aproximarse al análisis de la relación expansión residencial periurbana y crecimiento de población. De igual forma, se elaboró trabajo cartográfico el cual permitió identificar la localización de los conjuntos para comparar su dimensión respecto al área urbana municipal, y otras mediciones que ayudaron a caracterizar y analizar el crecimiento tanto urbano como poblacional.

\section{Resultados}

Para describir la evolución de los conjuntos urbanos y su influencia en el crecimiento de la población y el desarrollo de los espacios periurbanos en Calimaya, se consideró lo siguiente:

\section{Dinámica demográfica en el municipio de Calimaya}

El crecimiento de la población del municipio de Calimaya es una expresión de las transformaciones socio-territoriales derivadas de la expansión residencial periurbana y responde principalmente al fenómeno de migración de población que cambió su residencia al periurbano de este municipio en las últimas décadas.

Hasta 1990 el municipio presentó un crecimiento discreto de 24906 habitantes. A partir de la siguiente década la población, al igual que la expansión residencial periurbana, iniciaron un proceso acelerado de crecimiento. En 2000 el incremento fue de 41.3 por ciento respecto al anterior, ascendiendo a 35196 habitantes, hacia 2010 la población del municipio se incrementó a 47033 habitantes, 33.6 por ciento más respecto a 1990. En el año 2015 aumentó a 56574 habitantes, que representó 20.3 por ciento más que en 2010.

Así mismo, la tasa de crecimiento media anual que presentó la población de Calimaya muestra que los periodos de más crecimiento fueron de 1990-2000 con 3.33 por ciento y 2010-2015 de 3.76 por ciento, ambas por arriba de la tasa media anual de la Zona Metropolitana de Toluca (ZMT) y del Estado de México. Destaca la tasa de crecimiento media anual de 20102015 la cual fue de 3.76 por ciento por arriba de la ZMT de 0.73 por ciento y la del Estado de México de 1.54 por ciento (Tabla 1). 


\begin{tabular}{lcrrrrrr}
\hline $\begin{array}{l}\text { Tabla 1: Población total de Calimaya, ZMT y Estado de México y tasa media anual } \\
\text { de crecimiento (1990-2015) }\end{array}$ & \multicolumn{7}{c}{ TCMA } \\
\cline { 2 - 8 } Localización & 1990 & 2000 & 2010 & 2015 & 19902 & 20002 & 20102 \\
& 24906 & 35196 & 47033 & 56574 & 3.51 & 2.94 & 3.76 \\
\hline Calimaya & 1061065 & 1471146 & 1846116 & 1914892 & 3.32 & 2.29 & 0.73 \\
ZMT & 9802820 & 13096686 & 14995703 & 16187608 & 2.93 & 1.36 & 1.54 \\
Edo. México & \multicolumn{7}{c}{ Población } \\
\hline
\end{tabular}

Fuente: elaboración propia con base en los censos de población y vivienda 1990, 2000, 2010 y conteo 2015 del INEGI.

De esta manera, al conformarse el sector inmobiliario como eje de desarrollo de vivienda en el municipio, amplios sectores de la población urbana se vieron atraídos por las condiciones y beneficios que aquel ofrecía, generándose desplazamientos (movimientos migratorios ciudad-campo) que impactaron el crecimiento poblacional del municipio.

Otras condiciones intrínsecas y generadas, que permitieron el crecimiento demográfico son:

- La carretera estatal Toluca-Metepec-Tenango del Valle, la cual diariamente permite el traslado de bienes y personas a la capital del Estado, ya sea del propio Estado y la región, como de los estados del sur.

- La cercanía y accesibilidad con la ciudad de Toluca, Metepec y Distrito Federal que aumentó con la ampliación de la carretera estatal TolucaTenango del Valle y la autopista Lerma-Tenango del Valle.

- Las condiciones geográficas del terreno hacia el norte, oriente, sur y sureste de la cabecera municipal, con planicies y pendientes suaves que facilitan los asentamientos.

- La oferta y demanda de suelo y vivienda más económica que en la ciudad.

Esto contribuye a que el municipio concentre 0.37 por ciento de la población estatal, con 56574 habitantes y 2.95 por ciento de la ZMT en el año 2015.

\section{EXPANSIÓN URBANA Y PRODUCCIÓN DE CONJUNTOS URBANOS en el municipio de Calimaya}

Después de revisar las autorizaciones de conjuntos urbanos para la zona metropolitana de Toluca (ZMT), se elaboró una base de datos y se identificó que entre 1990 y 2015 se otorgaron 87 autorizaciones de conjuntos 
urbanos, de los cuales diez corresponden al municipio de Calimaya, lo que representa 11.49 por ciento del total de la ZMT (Tabla 2). Se aprecia que éstas han implicado la construcción de 10 conjuntos urbanos de diversa escala y tipología localizados en tres de siete localidades del municipio. Respecto a la distribución de los conjuntos urbanos en el territorio municipal, en un primer análisis, se verifica su ubicación en el espacio periurbano y destaca la producción de estos en las siguientes localidades: tres en Calimaya de Díaz González, seis en San Andrés Ocotlán y uno en La Concepción Coatipac (Tabla 3).

Tabla 2: Autorizaciones de conjuntos urbanos en Calimaya y ZMT de 1990-2015

\begin{tabular}{lrr}
\hline & \multicolumn{2}{c}{ Autorizaciones } \\
Área & Número & \% respecto al total \\
\hline Municipio de Calimaya & 10 & 11.49 \\
Zona Metropolitana de Toluca & 87 & 100 \\
\hline Fuente: elaboración propia con base en las estadísticas de conjuntos urbanos de la Secretaría de \\
Desarrollo Urbano del Estado de México. Disponible en & \\
http//www.edomex.gob.mx/sedur/stadisticas/conjuntos-urbanos
\end{tabular}

Tabla 3: Superficie urbanizada y conjuntos urbanos autorizados en las localidades de Calimaya de 1990-2015

\begin{tabular}{lrrrr}
\hline & \multicolumn{2}{c}{ Autorizaciones } & \multicolumn{2}{c}{ Superficie urbanizada } \\
\cline { 2 - 5 } \multicolumn{1}{c}{ Área } & Número & $\begin{array}{r}\text { \% respecto } \\
\text { al total }\end{array}$ & Hectáreas & $\begin{array}{r}\text { \% respecto } \\
\text { al total }\end{array}$ \\
\hline Calimaya de Díaz González & 3 & 30 & 205.0 & 53.45 \\
San Andrés Ocotlán & 6 & 60 & 146.55 & 38.10 \\
La Concepción Coatipac & 1 & 10 & 32.0 & 8.34 \\
Total del municipio & 10 & 100 & 383.57 & 100 \\
\hline Fony
\end{tabular}

Fuente: elaboración propia con base en las estadísticas de conjuntos urbanos de la Secretaría de Desarrollo Urbano del Estado de México. Disponible en http//www.edomex.gob.mx/sedur/stadisticas/conjuntos-urbanos

Un segundo análisis se refiere al crecimiento del área urbana de Calimaya generado por la construcción de conjuntos urbanos, y su impacto respecto a la superficie urbanizada preexistente y se tiene que: el crecimiento del área urbana del municipio de Calimaya por la construcción de conjuntos urbanos ha sido de 383.57 hectáreas, equivalente a 21.41 por ciento del total del área preexistente, y 28.16 por ciento del total del área del municipio. Las localidades con mayor impacto en el crecimiento del área urbana son Calimaya de Díaz González con 204.73 hectáreas, equivalente a 14.21 por ciento de la superficie urbanizada del municipio; San Andrés Ocotlán con 146.55 hectáreas, equivalente a 10.17 por ciento de la 
superficie urbanizada del municipio, y La Concepción Coatipac con sólo 31.92 hectáreas, equivalente a 2.21 por ciento de la superficie urbanizada del municipio (Tabla 3).

El impacto en la localidad de Calimaya de Díaz González ha sido fuerte no sólo por el tamaño de los conjuntos, sino por su localización y el breve periodo en que se han desarrollado, modificando su funcionamiento. $\mathrm{Si}$ bien esto resulta evidente, tratándose de la cabecera municipal, no ocurre lo mismo con las otras dos localidades, pues por ser más pequeñas, el impacto es mayor al provocar alteración en su funcionamiento, saturando la deficiente estructura y equipamiento, y sobre todo la afectación de la vida cotidiana de sus habitantes, generando áreas claramente diferenciadas entre poblaciones oriundas y nuevas lo cual promueve la segregación social en las áreas rurales.

\section{EVOLUCIÓN DE LOS CONJUNTOS URBANOS Y EL PERIURBANO de Calimaya}

En Calimaya, la autorización de los conjuntos urbanos inició en 1999 con el conjunto residencial Rancho el Mesón, de carácter exclusivo y cerrado, dirigido a estratos económicos altos (Figura 2), ubicado en suelos de producción agrícola de la localidad de San Andrés Ocotlán, cercanos a la vialidad principal Toluca-Calimaya. Este conjunto urbano que se vio como un hecho aislado y diferente al crecimiento tradicional urbano, fue el origen del proceso de expansión residencial en el espacio periurbano del municipio.

Posteriormente se autorizan y construyen dos conjuntos urbanos habitacionales en el año 2007, Villas del Campo I en el ex-rancho "Don Martín y la Esperanza" en la localidad de Calimaya de Díaz González y Residencial San Andrés en la localidad de San Andrés Ocotlán sobre la carretera Toluca-Tenango del Valle (Figura 3). A partir de estos, se inicia una carrera en la autorización y construcción de conjuntos urbanos.

Para el año 2009, se autorizan tres conjuntos más: Villas del Campo II e Ibérica en la localidad de Calimaya de Díaz González, y Valle del Nevado en la localidad de La Concepción Coatipac (Figura 4). Con la autorización de estos, ya sumaban seis conjuntos urbanos, los cuales evidenciaban la transformación del espacio rural, el cambio de usos de suelo agrícola a urbano y la reconfiguración urbana del municipio. 
Evolución de los conjuntos urbanos y su influencia en el crecimiento poblacional ... / M.L. GARCÍA-GONZÁLEZ et al.

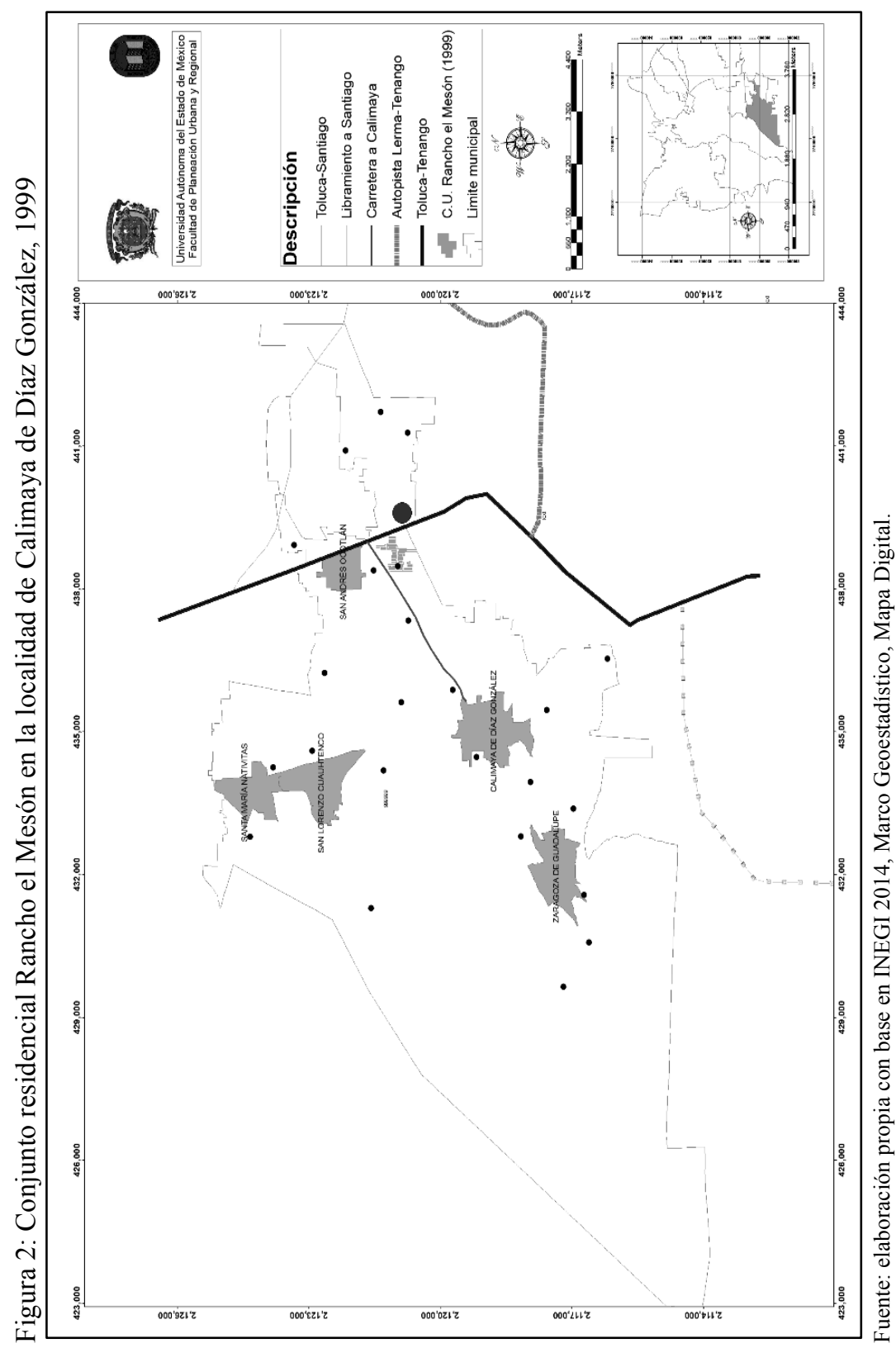







Evolución de los conjuntos urbanos y su influencia en el crecimiento poblacional ... / M.L. GARCÍA-GONZÁLEZ et al.

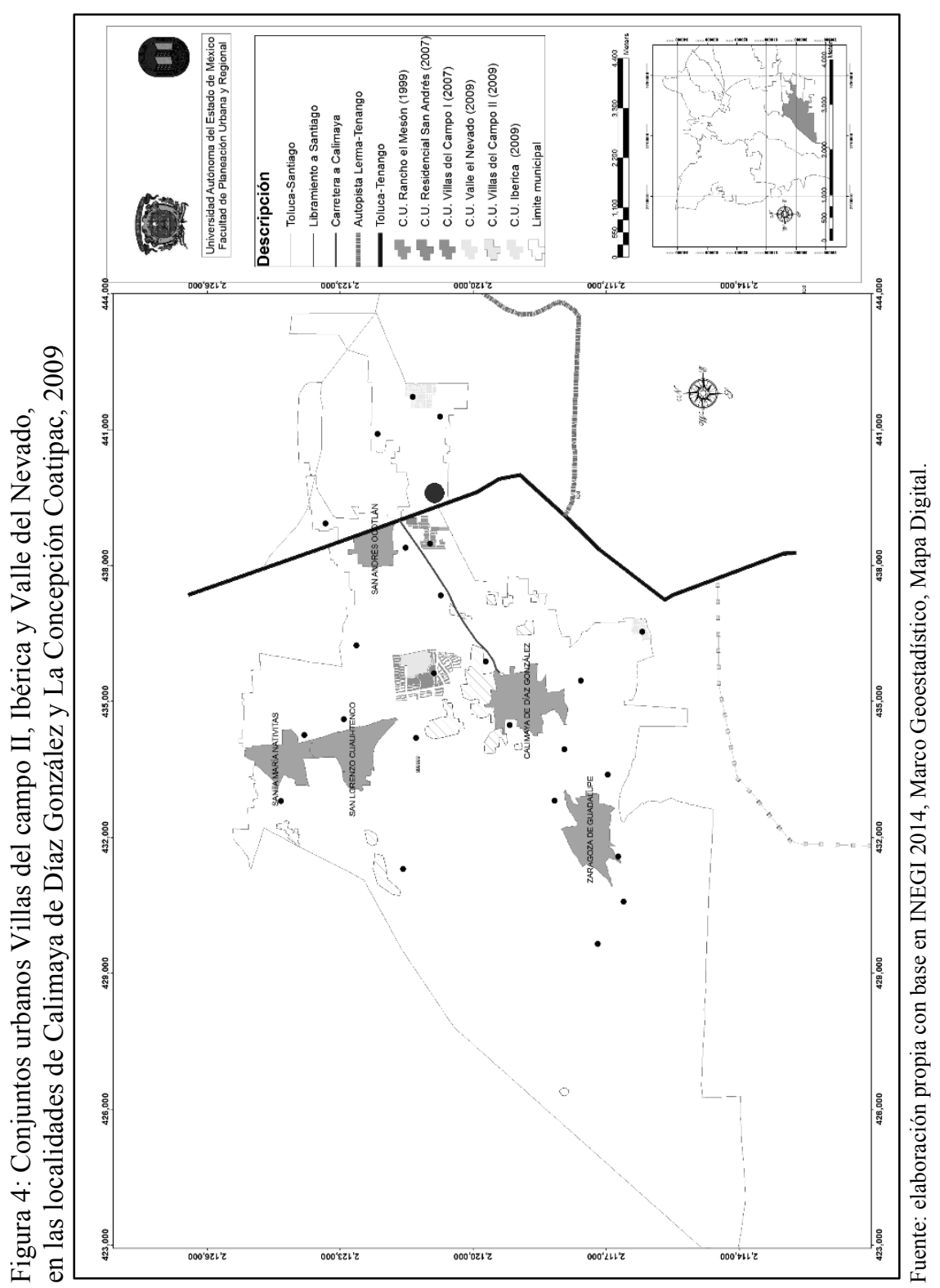


En 2010, se autorizan cuatro conjuntos urbanos más: Bosque de las Fuentes, Valle de las Fuentes, Hacienda de las Fuentes y Lomas Urbi, todos ellos en la localidad de San Andrés Ocotlán, los cuales se sumaron a los existentes, haciendo un total de diez conjuntos urbanos autorizados y construidos en sólo cuatro años (Figura 5). Estás nuevas urbanizaciones continuaron la transformación de los usos de suelo agrícola a urbano, e influyeron en la expansión residencial y conformación del espacio periurbano del municipio de Calimaya, consolidando a las localidades de Calimaya de Díaz González, San Andrés Ocotlán y la Concepción Coatipac como zonas para la construcción de vivienda para sectores sociales de ingresos altos, medio altos, medios y de interés social.

Dentro del municipio destaca la producción de vivienda residencial y residencial-media a través de la figura del conjunto urbano cerrado, en las localidades de Calimaya de Díaz González y San Andrés Ocotlán, ubicados en la cercanía de la vialidad Toluca-Calimaya y Toluca-Tenango y el conjunto abierto de interés social en la periferia de La Concepción Coatipac, alejado de la vialidad Toluca-Tenango y Toluca-La Concepción Coatipac.

Respecto a su evolución, se observa que en el año 2010 (Figura 6) se consolidan estas localidades como una zona para el desarrollo de conjuntos urbanos, para población de ingresos altos, medios, y de interés social.

En suma, la expansión de los conjuntos urbanos y la formación del espacio periurbano se localizaron al noreste de la cabecera municipal en Calimaya de Díaz González, al sur y noreste de la localidad de San Andrés Ocotlán y al oriente de la localidad de La Concepción Coatipac (Figura 6).

Con la autorización y localización de los conjuntos urbanos, no solo se fomenta el cambio de uso de suelo agrícola a urbano, la reconfiguración de la estructura urbana, la formación del espacio residencial periurbano, el crecimiento rápido de la población y el movimiento pendular de los nuevos habitantes, congestionando las vialidades e incrementando la contaminación atmosférica, sino además, se promueve la segregación social y residencial en el territorio. Esta situación se relaciona directamente con la escala de intervención medida a través de la cantidad de viviendas, y su tipo por conjunto y localización, lo que implicó un tercer análisis.

Mientras que en la cabecera municipal Calimaya de Díaz González, se desarrollaron tres conjuntos urbanos con un total de 4144 nuevas viviendas, en la localidad de San Andrés Ocotlán, se han producido seis conjuntos urbanos con 4035 viviendas, y en La Concepción Coatipac, 2783 nuevas viviendas distribuidas en solamente un conjunto urbano (Tabla 4). 
Evolución de los conjuntos urbanos y su influencia en el crecimiento poblacional ... / M.L. GARCÍA-GONZÁLEZ et al.




Figura 6: Evolución de conjuntos urbanos según su ubicación y autorización en Calimaya de 1999 a 2015

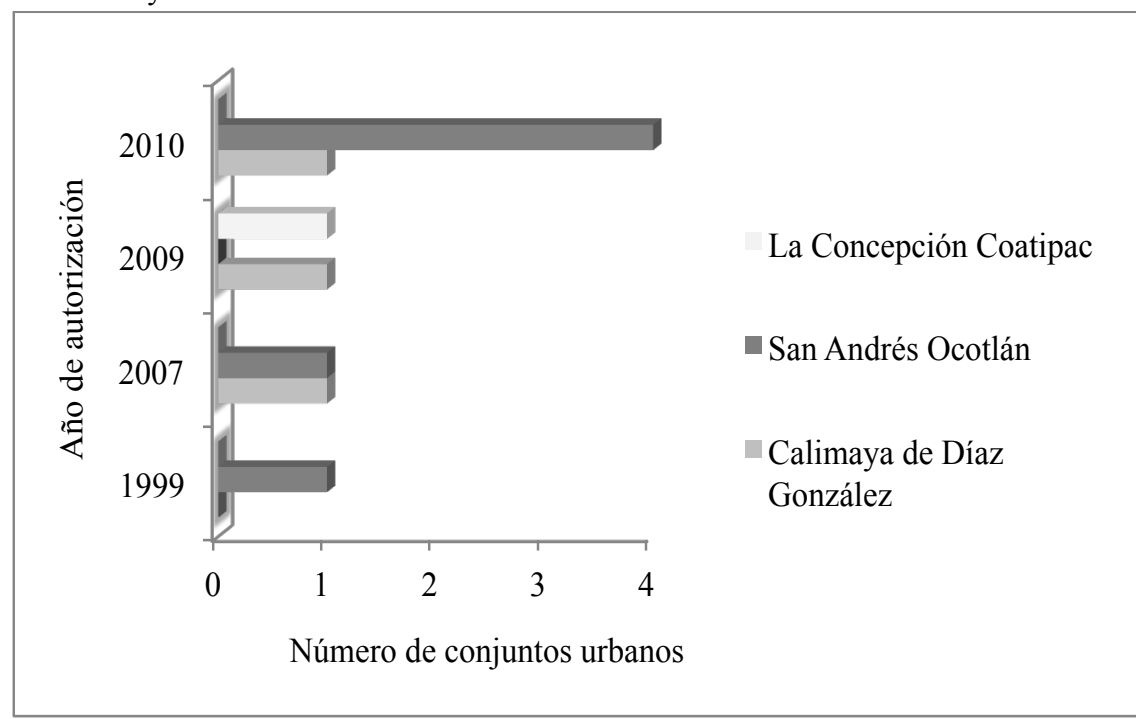

Fuente: elaboración propia con base en información de GACETAS del GEM, SDUEM.

Tabla 4: Evolución de la producción de vivienda residencial según localización de 1990 a 2015

\begin{tabular}{lcrrrrr}
\hline \multirow{2}{*}{ Localidad } & $\begin{array}{c}\text { Conjuntos } \\
\text { urbanos }\end{array}$ & 1999 & 2007 & 2009 & 2010 & Total \\
\hline Calimaya de Díaz González & 4 & 0 & 1313 & 1892 & 2060 & 5265 \\
San Andrés Ocotlán & 5 & 388 & 121 & 0 & 1776 & 2285 \\
La Concepción Coatipac & 1 & 0 & 0 & 2783 & 0 & 2783 \\
Total & 10 & 388 & 1434 & 4675 & 3836 & 10333 \\
\hline
\end{tabular}

Fuente: elaboración propia con base en información de GACETAS del GEM, SDUEM.

El crecimiento de vivienda de mayor impacto para el municipio fue en el año 2009, con la construcción 4675 nuevas viviendas, y el de menor impacto fue en 1999 con solo 388. El efecto que se tiene en el municipio con la construcción de 10 conjuntos urbanos y la producción de 10333 viviendas es muy fuerte, ya que unicamente en cuatro años se produjeron 171.08 por ciento de las viviendas existentes en 1995, que correspondía a 6040 viviendas.

Debido a la oferta de vivienda por los conjuntos urbanos, se llevó a cabo la caracterización de éstos, con base en la tipología establecida en el Código 
Financiero del Estado de México y municipios (GEM, 2002b: Artículo 3, inciso XL).

Como se observa en la Tabla 5 en la producción de conjuntos urbanos destaca la tipología de conjuntos cerrados con 90 por ciento en las localidades de Calimaya de Díaz González y de San Andrés Ocotlán, concentrando el mayor número de viviendas para la población de recursos altos y medios, y sólo se tiene un conjunto urbano abierto en la localidad de La Concepción Coatipac, para población de escasos recursos.

Tabla 5: Conjuntos urbanos según tipología y localización de 1990 a 2015

\begin{tabular}{lrrrr}
\hline \multicolumn{1}{c}{ Localidad } & $\begin{array}{r}\text { Conjunto } \\
\text { urbano cerrado }\end{array}$ & $\begin{array}{r}\text { \% respecto } \\
\text { al total }\end{array}$ & $\begin{array}{r}\text { Conjunto } \\
\text { Urbano abierto }\end{array}$ & $\begin{array}{r}\% \text { respecto } \\
\text { al total }\end{array}$ \\
\hline Calimaya de Díaz González & 3 & 30 & 0 & 0 \\
San Andrés Ocotlán & 6 & 60 & 0 & 0 \\
La Concepción Coatipac & 0 & 0 & 1 & 10 \\
Total & 9 & 90 & 1 & 10 \\
\hline
\end{tabular}

Fuente: elaboración propia con base en información de GACETAS del GEM, SDUEM, GEM y registro de campo.

Cabe mencionar que, mientras que los conjuntos urbanos cerrados están construidos en las inmediaciones o a las orillas de las principales vialidades Toluca-Calimaya y Toluca-Tenango del Valle, el conjunto urbano abierto de interés social se haya alejado de la localidad urbana y de la vialidad Toluca-Tenango del Valle. Estas nuevas formas de producir vivienda promueven en el territorio municipal: la fragmentación y segregación socioespacial, además de saturar las vialidades y hacer más complicada la vida de los nuevos habitantes de los conjuntos urbanos, ya que se deben mover a grandes distancias para trabajar, ir a la escuela o realizar sus compras y para la administración municipal imposibilidad de atender las necesidades de los nuevos habitantes, debido a sus escasos recursos.

En relación a su tipología, de acuerdo con lo establecido en el Código Financiero del Estado de México y municipios (GEM, 2002b: Artículo 3, inciso XL), la Tabla 6 muestra que la producción de vivienda en el municipio está dirigida en su mayoría a estratos sociales altos, medio-altos y medios, de tal forma que las tipologías: residencial y mixto-residencial cuenta con 1932 viviendas, la mixto-media y media con 5618 viviendas, representando 73.07 por ciento del total de viviendas localizadas en las localidades de Calimaya de Díaz González y San Andrés Ocotlán. La producción de vivienda de interés social fue de 2788 viviendas, localizadas en La Concepción Coatipac, a mayor distancia de la cabecera municipal 
y centro urbano. De esta manera, Calimaya se proyecta como una nueva opción para el desarrollo de proyectos de vivienda para los sectores económicos altos y medios.

Tabla 6: Vivienda según tipología y localización de 1990 a 2010

\begin{tabular}{lrrrrr}
\hline \multirow{2}{*}{ Localidad } & \multicolumn{5}{c}{ Vivienda según tipología } \\
\cline { 2 - 6 } & Residencial & $\begin{array}{r}\text { Mixto } \\
\text { residencial }\end{array}$ & $\begin{array}{r}\text { Mixto } \\
\text { medio }\end{array}$ & Medio & $\begin{array}{r}\text { Interés } \\
\text { social }\end{array}$ \\
\hline Calimaya de Díaz González & 0 & 93 & 1220 & 3,952 & 0 \\
San Andrés Ocotlán & 388 & 1451 & 0 & 446 & 0 \\
La Concepción Coatipac & 0 & 0 & 0 & 0 & 2783 \\
Total & 388 & 1544 & 1220 & 4398 & 2783 \\
\hline
\end{tabular}

Fuente: Elaboración propia con base en GACETAS del GEM, SDUEM, GEM y registro de campo.

En cuanto a la participación de las empresas inmobiliarias en la expansión residencial, construcción del espacio periurbano y crecimiento de población, cuarta parte de la investigación (Figura 7). Se tiene que GEO edificaciones S.A. de C.V es la empresa inmobiliaria que tiene la mayor presencia por el número de autorizaciones, con proyectos de conjuntos urbanos cerrados de tipo residencial medio, seguida de la empresa Proyectos Inmobiliarios de Culiacán S.A. de C.V, con vivienda de tipo media e interés social. El resto de empresas inmobiliarias contribuye con sólo una autorización de los conjuntos urbanos.

Figura 7: Autorizaciones según desarrolladora inmobiliaria de 1990 a 2015

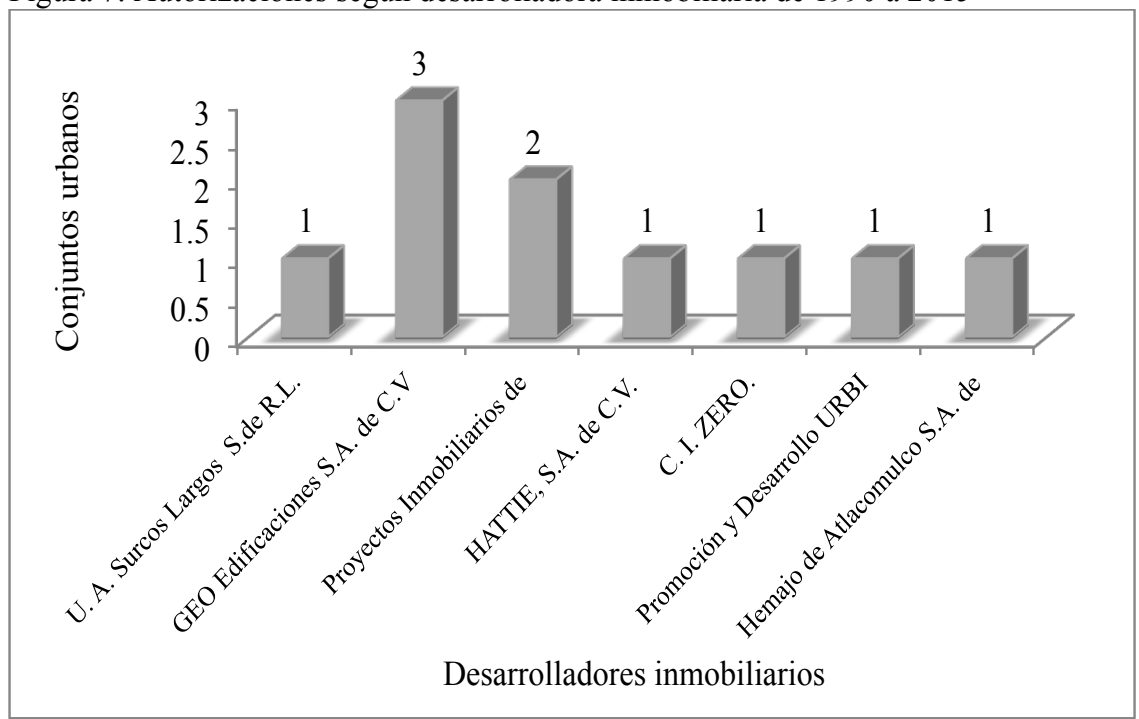

Fuente: elaboración propia con base en información de GACETAS del GEM, SDUEM, GEM y registro de campo. 
Respecto al número de viviendas construidas por desarrolladora inmobiliaria en el periodo de estudio (Figura 8), destaca GEO edificaciones S.A. de C.V. por la construcción de 4955 viviendas (47.95 por ciento), seguida de Proyectos Inmobiliarios de Culiacán S.A. de C.V. con 3093 viviendas (29.94 por ciento) y Hemajo Atlacomulco con sólo 121 viviendas (1.17 por ciento) construidas durante el periodo de estudio.

Figura 8: Viviendas por desarrolladora inmobiliaria en Calimaya de 1990 a 2010

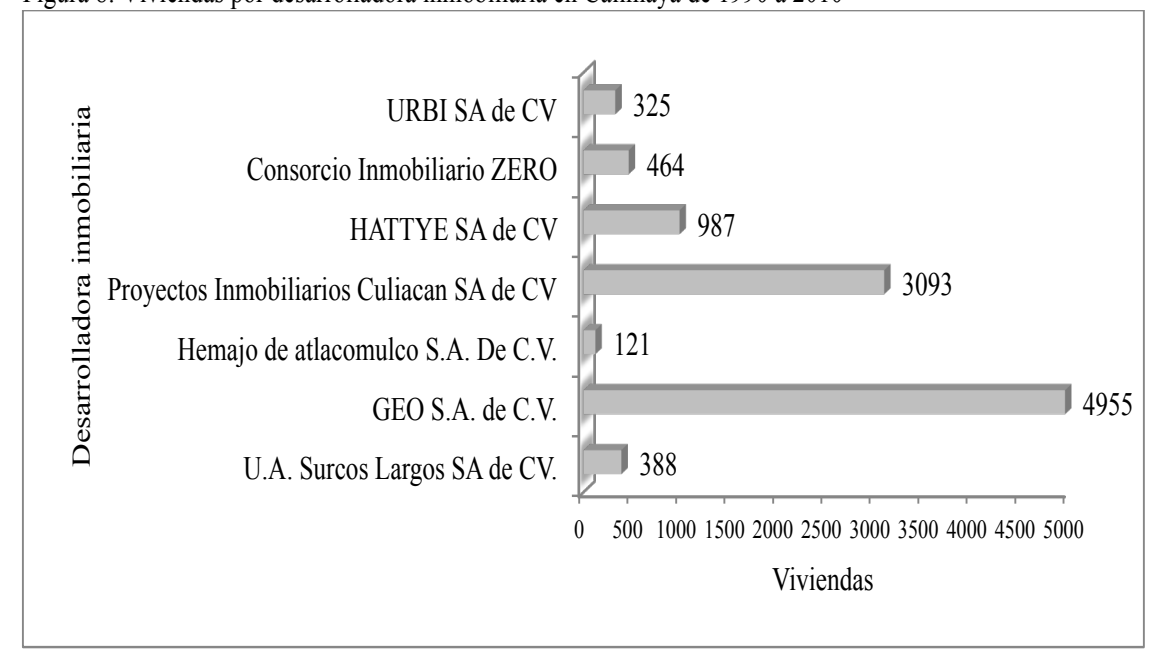

Fuente: elaboración propia con base en autorizaciones de construcción GACETAS, SDUEM, GEM.

\section{Conclusiones}

La expansión residencial y el incremento poblacional en el municipio de Calimaya responde a diversos factores, entre los que destacan su posición geográfica e interconexión en el intercambio de bienes y personas con la capital de Estado y el sur de la región, la disponibilidad y bajo costo de suelo urbanizable, la apertura a desarrolladores de vivienda privados y la demanda de vivienda y seguridad, a la cual da respuesta el gobierno a través de la política de financiamiento de vivienda. Ésta se manifiesta en la forma de ocupación y organización del espacio periurbano, los nuevos modelos habitacionales en la modalidad de conjuntos urbanos; cerrados y abiertos, de tipo: residencial, residencial mixto y de interés social. Surgen y se reproducen ante la flexibilidad de los instrumentos de planeación, que permitieron la construcción dispersa en zonas poco accesibles a los centros de población. Con una tendencia a extenderse sin control en las localidades de Calimaya de Díaz González y San Andrés Ocotlán, en las áreas agríco- 
las de alta y mediana productividad que las circundan y donde se convive con las actividades extractivas de materiales pétreos, en detrimento de la calidad de vida de los nuevos habitantes de estas formas de urbanización.

Ahora bien, el desarrollo de conjuntos urbanos en la periferia del municipio inicia en 1999 con la construcción de un conjunto urbano residencial cerrado. De 2007 a 2010, se autorizaron nueve conjuntos urbanos más, representando un incremento de 90 por ciento, localizados tres en Calimaya de Díaz González en una superficie de 205 hectáreas; seis en San Andrés Ocotlán en 146.5 hectáreas y uno en la Concepción Coatipac en 32 hectáreas, transformando 383.57 hectáreas de suelo de alta y mediana productividad agrícola, las cuales representan 8.3 por ciento del territorio municipal.

En cuanto a la magnitud, entendida como la cantidad de vivienda producida, en diez conjuntos urbanos se construyeron 10333 viviendas, más del cien por ciento respecto a las existentes, trastocando la funcionalidad municipal, tanto de la población como del gobierno, donde la demanda de servicios supera los beneficios económicos de los impuestos en el gobierno municipal, el cual se ve incapacitado para atender las demandas de los nuevos habitantes. El modelo de urbanización privado corresponde con el periodo de gobierno federal y Estatal en el que se tuvo mayor apertura a la iniciativa privada en materia de vivienda. Fenómeno que reconfigura la estructura urbana y promueve la urbanización dispersa, a saltos y promueve la segregación socio-territorial y contaminación, en detrimento del ambiente y de la calidad de vida para toda la población del municipio y de la región.

El crecimiento de la población del municipio como efecto de la construcción de vivienda residencial y de interés social observó un cambio importante, al pasar de 24906 habitantes en 1990 a 56574 habitantes para 2015. Esta población requiere servicios como tratamiento de las aguas residuales, residuos sólidos urbanos, seguridad, salud y educación entre otros, evidenciando las incapacidades de la administración pública municipal para atenderlos.

Los movimientos pendulares de esta población ocasionan congestionamiento de las vialidades Toluca-Calimaya, Toluca-Tenango del Valle y Tenango del Valle- Lerma, incorporando contaminantes de efecto invernadero en detrimento de la calidad de vida de la población de ésta zona.

Destacan los actores que influyeron en estos procesos, de las diez desarrolladoras inmobiliarias; GEO edificaciones S.A. de C.V. participaron con tres conjuntos urbanos cerrados de viviendas de tipo residencial mixto y 
medio, Proyectos Inmobiliarios de Culiacán S.A. de C.V. con dos conjuntos, uno abierto y uno cerrado, con viviendas de tipo medio y de interés social. El resto de las desarrolladoras participó con un proyecto de vivienda.

Así pues, este nuevo modelo de urbanización en Calimaya no es ajeno a una realidad globalizada, que refuerza estándares de convivencia y consumo dirigidos por el capital privado y sus intereses, aunado a factores como la búsqueda de seguridad, estatus social sin identidad, y calidad de vida referida a un entorno ambiental saludable y la flexibilidad de los instrumentos de planeación urbana estatal y municipal. De persistir la tendencia de crecimiento urbano y de población en el municipio de Calimaya, se acrecentará el patrón urbano de baja densidad y la pérdida de suelo con aptitudes para el uso agrícola, lo que agudizará el consumo de recursos como el hídrico y alimentos básicos agrícolas y la gestión de los Residuos Sólidos Urbanos, aguas residuales y contaminación del aire. Lo anterior pone en evidencia a los gobiernos federal, estatal y municipal en el cumplimiento de la normatividad urbana.

\section{REFERENCIAS BIBLIOGRÁFICAS}

Adell, G., 1999, Theories and Models of the Peri-Urban Interface: A Changing Conceptual Landscape, Ouput 1, Research Projet, Strategis Environmental Planning and Management for de Peri-Urban InterfaceResarch Proyect. Development Planning Unit. University College, DPU. Londres.

Aguilar, G. A., 2006, Las grandes aglomeraciones y su periferia regional, experiencias en Latinoamérica y España. Editorial Miguel Ángel, Porrúa. México.

Allen, A., 2003, La interface periurbana como escenario de cambio y acción hacia la sustentabilidad del desarrollo. Cuaderno del Cendes, Caracas.

Ávila, S. H., 2001, "Ideas y planteamientos teóricos sobre los territorios pertúrbanos. Las relaciones campo-ciudad en algunos países de Europa y América”, en Investigaciones geográficas, núm. 45., Boletín del instituto de geografía, UNAM, México.

Ávila, S.H., 2009, "Periurbanización y espacios rurales en la periferia de las ciudades", en Estudios Agrarios, Procuraduría Agraria, México.

Barsky, A., 2005, "El periurbano productivo, un espacio en constante transformación. Introducción al estado de debate, con referencias al caso de Buenos Aires", en Scripta Nova, Revista electrónica de Geografía y Ciencias Sociales, vol. IX, núm.194, Universidad de Barcelona.

Berry, B., 1976, “The counter urbanization process: urban America since 1970”, in Berry, B. (ed.) Urbanization and counterurbanization, Sage: Beverly Hills. 
Capel, H., 1994, "Las periferias urbanas y la geografía. Reflexiones para arquitectos", en Anthropos, núm. 43, suplemento La geografía hoy. Textos, historia y documentación, Material de trabajo intelectual, Barcelona.

Cabrales, B. y Canosa Z., E., 2001, “Segregación residencial y fragmentación urbana: los fraccionamientos cerrados en Guadalajara", en Espiral: Estudios sobre estado y sociedad, vol. 7, núm. 20. Guadalajara.

Duhau, E., 2008, “Los nuevos productores del espacio habitable”, en Ciudades, vol. 20 , núm. 69 .

Garay, A., 2001, Dimensión territorial de lo local, Curso de Desarrollo Local de Áreas Metropolitanas. Modulo 2. Instituto del Conurbano, Universidad Nacional de General de Sarmiento.

GEM, 2002a, Código Administrativo del Estado de México. Gaceta del Gobierno del Estado de México del 13 de marzo del 2002. Toluca, México.

GEM, 2002b, Código Financiero del Estado de México y Municipios. Gaceta del Gobierno del Estado de México del 13 de marzo, Toluca, México.

GEM, 2002c, Reglamento del Libro Quinto del Código Administrativo. Gaceta del Gobierno del Estado de México del 13 de marzo, Toluca, México.

GEM, s/f, Estadísticas de Conjuntos Urbanos. Secretaría de Desarrollo Urbano, disponible en http://portal2.edomex.gob.mx/sedur/informacion_de_interes/conjuntos_urbanos/index.htm

GEM, 2003, Plan de Desarrollo Urbano Municipal. Honorable Ayuntamiento Constitucional de Calimaya.

GEM, 2007, Modificación del Plan Municipal de Desarrollo Urbano Municipal. Honorable Ayuntamiento Constitucional de Calimaya.

GEM, 2011, Plan de Desarrollo Urbano Municipal. Honorable Ayuntamiento Constitucional de Calimaya.

GEM, 2013, Plan de Desarrollo Urbano Municipal. Honorable Ayuntamiento Constitucional de Calimaya.

INEGI, 1990, Censo general de población y vivienda, 1990, Aguascalientes, México,

INEGI, 2000, XII Censo general de población y vivienda, 2000. Tabulados básicos, Aguascalientes, México.

INEGI, 2005, II Conteo de población y vivienda, 2005. Tabulados básicos, Aguascalientes, México.

INEGI, 2010, XIII Censo general de población y vivienda, 2000. Tabulados básicos, Aguascalientes, México.

Iracheta, C. y Pedrotti, C. I., 2013, "Expansión metropolitana y producción habitacional en la Zona Metropolitana de Toluca", en Medina, C. S. (coordinadora), Contribuciones al estudio de las ciudades, el Estado de México y el suelo urbano. Colegio Mexiquense, Zinacantepec, México.

Kayser, B., 1990, La renaissance rurale: sociologie des campagnes du monde occidental, Armand Colin. París. 
Puebla, G., 2004, “Aproximaciones al concepto de periurbano”, mimeo, Caseros: Cátedra de Gestión Local, Universidad Nacional de Tres de Febrero.

Zarate, M., 1991, El espacio interior de la ciudad, Madrid, España.

\section{RESUMEN CURRICULAR DE LOS AUTORES}

\section{María de Lourdes García González}

Maestra en Ciencias Ambientales y Doctorante en Urbanismo. Publicaciones recientes: La introducción de la temática ambiental como un elemento categórico en la formación del Licenciado en Diseño Gráfico e Industrial, El diseño en la construcción de la ciudad para todos, compiladores Jesús Enrique de Hoyos Martínez, Liliana, Romero Guzmán, José de Jesús Jiménez Jiménez y Alberto Álvarez Vallejo, "Expansión metropolitana de Toluca: caso de estudio municipio de Calimaya, México", en Quivera, vol. 17, núm 1, enero-junio, 2015, Adscripción: Facultad de Planeación Urbana y Regional de la UAEM, profesora de Asignatura.

Dirección electrónica: ma_lulu11@yahoo.com.mx

\section{Fermín Carreño Meléndez}

Doctor en Urbanismo. Publicaciones recientes: Coordinador del Libro Sustentabilidad productiva sectorial 2015. Autor del capitulo de "Neza II riesgo y Responsabilidad Gubernamental", en Crisis del modelo fosilista y la insustentabilidad ¿O debacle de la civilización accidental, 2015. Adscripción: Coordinador del Centro de Estudios e Investigación en Desarrollo Sustentable, UAEM.

Dirección electrónica: fermin_carreno@yahoo.com.mx

\section{Alfonso Mejía Modesto}

Doctor en Ciencias Políticas y Sociales. Publicaciones recientes: Artículo: "Estimación del impacto de los cambios de la fecundidad en la dinámica demográfica del Estado de México a nivel municipal", en Papeles de Población núm. 84. abril-junio 2015, Centro de Investigación y Estudios Avanzados de la Población UAEM; capítulo de libro: "Cambios en la formación familiar a inicios del siglo XXI en México", en Los hogares y las familias en el Estado de México, noviembre 2015. Adscripción: Centro de Investigación de Investigación y Estudios Avanzados de la Población de la UAEM.

Dirección electrónica: mejiaalfonso@yahoo.com.mx 